\title{
Utilisation des écumes de sucrerie pour la désulfuration des fumées
}

\author{
J.-C. Dolignier et G. Martin ${ }^{1}$ \\ 1 Institut français du pétrole, 1 et 4, avenue de Bois-Préau, 92852 Rueil-M almaison Cedex - France
}

\begin{abstract}
Résumé - Des études ont été réalisées par l'Institut français du pétrole et Babcock Entreprise pour montrer que l'écume de sucrerie pouvait être utilisée comme agent de désulfuration dans la chaudière Aude, un nouvel équipement de combustion permettant l'emploi de combustibles à haute teneur en soufre. Les tests ont été effectués en laboratoire et sur une chaudière semi-industrielle de $10 \mathrm{MW}$. L'écume de sucrerie donne des rendements de désulfuration supérieurs à ceux obtenus avec des calcaires naturels finement broyés. De plus, l'azote contenu dans l'écume permet de réduire les émissions de $\mathrm{NO}_{\mathrm{x}}$ selon le mécanisme de la réduction sélective non catalytique. Les utilisateurs de combustibles à haute teneur en soufre, qui devront faire face à une législation de plus en plus sévère, pourraient réduire leur coût annuel d'absorbants en utilisant l'écume de sucrerie. Par ailleurs, les producteurs de sucre auraient l'opportunité de mettre en place une nouvelle filière de valorisation pour leurs écumes. La teneur en eau élevée de l'écume n'autorise pas un transport sur de longues distances et des calculs ont été effectués pour définir la distance à partir de laquelle l'écume devient moins rentable que le calcaire.
\end{abstract}

Mots-clés : désulfuration, sulfatation, dénitrification, chaudière, fioul lourd, combustion.

\begin{abstract}
Using Sugar Factory Carbonatation Lime for Flue-Gas Desulfurization - Studies have been carried out by the Institut français du pétrole and Babcock Entreprise to demonstrate that sugar factory carbonatation lime can be used as a desulfurization agent in the Aude boiler, which is a new combustion apparatus designed to use high sulfur fuels. Tests have been conducted both in the laboratory and on a near-industrial-scale $10 \mathrm{MW}$ boiler. Sugar factory carbonatation lime provides greater desulfurization efficiency than that obtained using finely crushed natural limestone. Furthermore, the nitrogen contained in the carbonatation lime reduces $\mathrm{NO}_{x}$ emissions through selective non-catalytic reduction. Those who use high sulfur content fuels, and who are faced with increasingly stringent legislation, could reduce their annual outlays for absorbents by using sugar factory carbonatation lime. In addition, sugar producers could open up a new channel for using their carbonatation lime. The high water content of carbonatation lime rules out long distance transport. Calculations have been carried out to indicate at what distance carbonatation lime becomes more expensive than limestone.
\end{abstract}

Keywords: desulfurization, sulfation, denitrification, boiler, heavy fuel oil, combustion.

\section{IN TRO DUCTION}

La législation sur les émissions de polluants des installations de combustion fixes de plus de $50 \mathrm{MW}$, utilisant des combustibles liquides, limite les rejets de $\mathrm{SO}_{2}$ à $1700 \mathrm{mg} / \mathrm{Nm}^{3}$ de fumées sèches à $3 \% \mathrm{O}_{2}$. Les utilisateurs de fiouls lourds doivent donc, en absence de dispositif de désulfuration, orienter leur approvisionnement vers les produits à très basse teneur en soufre, plus onéreux et disponibles en quantité plus faible sur le marché. La chaudière Aude (AUtoDépolluante), développée conjointement par l'Institut français du pétrole (IFP) et Babcock Entreprise, a été conçue pour répondre aux exigences de la législation, maintenant ainsi des débouchés aux produits du fonds du baril dans le domaine de la production d'énergie. Dans la chaudière Aude, les émissions de dioxyde de soufre sont contrôlées par injection d'un absorbant alcalin pulvérulent. La désulfuration est opérée à haute température, au-delà de $850^{\circ} \mathrm{C}$, dans un espace isotherme spécialement aménagé en aval du foyer de combustion. Cette technique est particulièrement attractive dans le cas des installations de moins de 
$300 \mathrm{MW}_{\text {th }}$, qui ne peuvent supporter les investissements liés aux traitements par voie humide.

Les études réalisées par l'IFP et Babcock Entreprise montrent que l'injection d'écume de sucrerie permet d'obtenir des rendements de désulfuration supérieurs à ceux obtenus avec les calcaires broyés du commerce. Elles montrent également que le contenu azoté de l'écume de sucrerie permet de réduire les émissions de $\mathrm{NO}_{\mathrm{x}}$. Ces études ont été effectuées en laboratoire, sur une installation de $250 \mathrm{~kW}$, et sur une chaudière semi-industrielle de $10 \mathrm{MW}$. Ce projet a reçu l'appui de la Commission européenne dans le cadre du programme Life.

\section{LA CHAUDIÈRE AUDE}

La chaudière Aude [1] possède l'architecture classique des installations de combustion de fioul lourd. Elle est constituée de quatre éléments (fig. 1) :

- la chambre de combustion ;

- la chambre de désulfuration et de dénitrification;

- la section d'échange thermique par convection ;

- le dépoussiéreur.

Le dioxyde de soufre est fixé par un absorbant alcalin pulvérulent introduit à l'entrée de la chambre de désulfuration. L'absorbant est récupéré par un dépoussiéreur électrostatique ou à manches, puis partiellement recyclé vers la chambre de désulfuration pour limiter la consommation de produit frais et le volume de résidu à éliminer.
Lorsque les performances des brûleurs ne permettent pas de respecter les normes relatives aux rejets de $\mathrm{NO}_{\mathrm{x}}$, deux types de traitement de fumées peuvent être installés :

- l'étagement du combustible (reburning);

- la réduction sélective non catalytique, par injection d'urée ou d'ammoniaque.

La chaudière Aude se distingue des équipements classiques par:

- une chambre de combustion plus volumineuse, pour accroître l'extraction de chaleur et limiter la production de $\mathrm{NO}_{\mathrm{x}}$ thermique ;

- la présence de la chambre de désulfuration et de dénitrification, isotherme et dimensionnée pour assurer un mélange de qualité entre les effluents gazeux et l'absorbant;

- un dépoussiéreur de grande capacité, c'est-à-dire supportant des charges en solides de plusieurs dizaines de grammes par normal mètre cube $\left(\mathrm{g} / \mathrm{Nm}^{3}\right)$;

- une zone d'échange de chaleur par convection dimensionnée pour opérer avec des concentrations en solides de quelques dizaines de grammes par normal mètre cube ;

- des dispositifs de stockage et d'acheminement de l'absorbant.

\section{DÉSULFURATION}

La plupart des oxydes métalliques permettent de fixer le dioxyde de soufre [2], mais les absorbants calciques, le calcaire ou la chaux, sont généralement préférés pour des

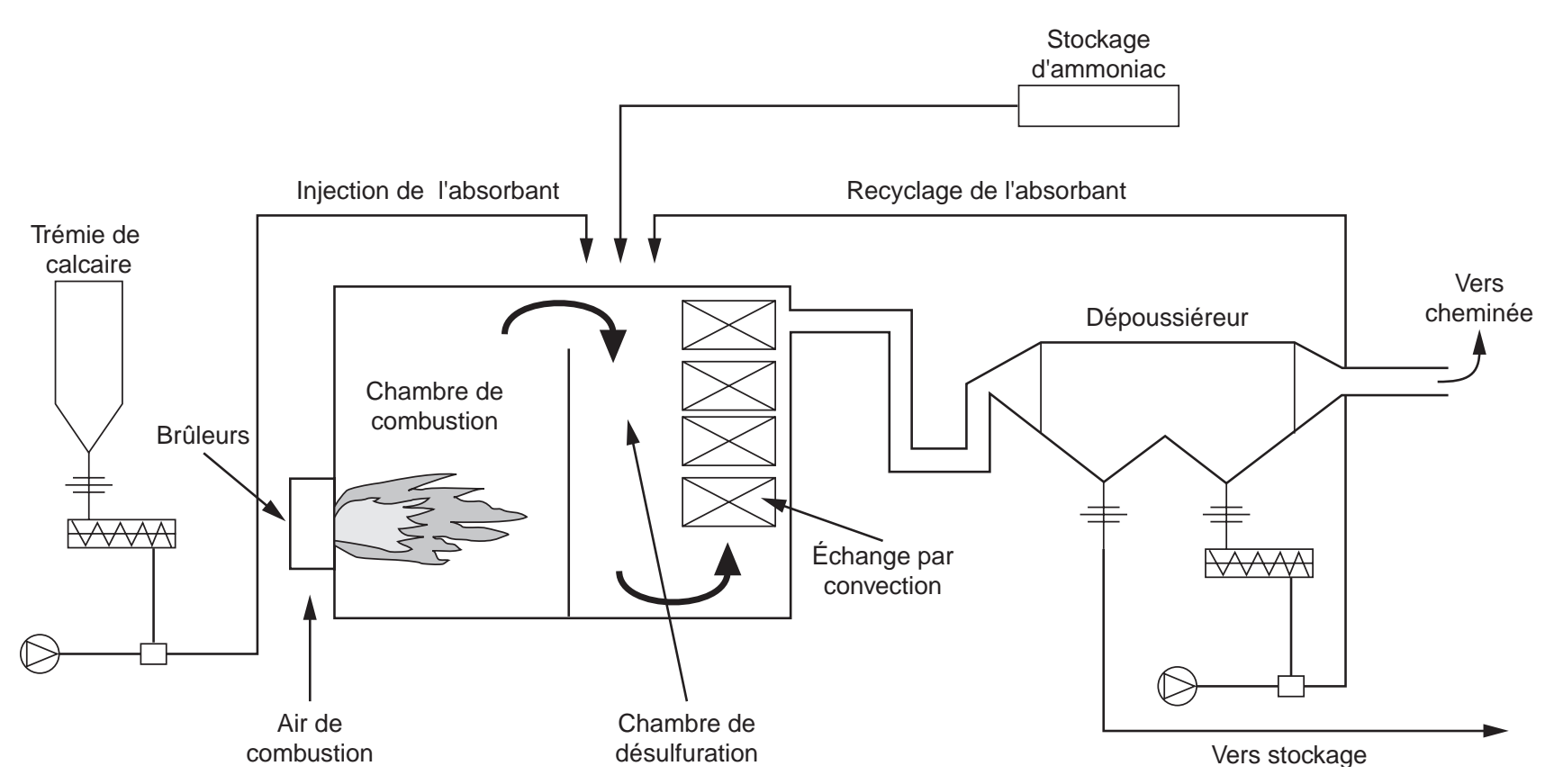

Figure 1

La chaudière Aude.

Aude Boiler. 
raisons économiques. Les résidus calcaires industriels peuvent également être utilisés. Certains travaux [3] ont par exemple montré que l'écume de sucrerie avait de bonnes aptitudes à la désulfuration. À haute température, au-delà de $850^{\circ} \mathrm{C}$, le dioxyde de soufre est fixé en deux étapes :

- la calcination, qui produit un oxyde de calcium poreux car le volume molaire du $\mathrm{CaO}$ est inférieur à celui du $\mathrm{CaCO}_{3}$ ou du $\mathrm{Ca}(\mathrm{OH})_{2}$;

- La sulfatation de l'oxyde de calcium.

Le schéma réactionnel global est donné ci-dessous :

$$
\begin{gathered}
\mathrm{CaCO}_{3} \rightarrow \mathrm{CaO}+\mathrm{CO}_{2} \text { ou } \mathrm{Ca}(\mathrm{OH})_{2} \rightarrow \mathrm{CaO}+\mathrm{H}_{2} \mathrm{O} \\
\mathrm{CaO}+\frac{1}{2} \mathrm{O}_{2}+\mathrm{SO}_{2} \rightarrow \mathrm{CaSO}_{4}
\end{gathered}
$$

La sulfatation de l'oxyde de calcium fait intervenir plusieurs étapes élémentaires : la diffusion du dioxyde de soufre à l'intérieur de la particule poreuse, l'adsorption à l'interface gaz-solide, la diffusion à travers la couche de sulfate en construction, puis la sulfatation [4]. La surface de réaction disponible augmente lors de la calcination mais est affectée par le frittage, processus thermiquement activé, responsable d'une coalescence des grains élémentaires qui forment la particule [5]. Le rendement de désulfuration dépend des propriétés de l'absorbant, porosité et surface spécifique, de la température et du temps de séjour.

\section{DÉSULFURATION PAR L'ÉCUME DE SUCRERIE : ÉTUDE EN LABORATO IRE}

\subsection{Montage expérimental}

Le réacteur de l'IFP (fig. 2) a été construit pour simuler la chambre de désulfuration de la chaudière Aude. Il s'agit d'un réacteur isotherme opérant en lit entraîné, assurant une circulation verticale descendante des gaz et des solides. L'introduction des gaz et des solides dans le réacteur a été définie de manière à atteindre très rapidement des conditions d'écoulement piston.

Les gaz chauds sont produits par un brûleur de gaz naturel et le $\mathrm{SO}_{2}$ est injecté à la sortie du four. L'isothermicité du réacteur est garantie par une isolation efficace et un chauffage électrique de la paroi. La température est mesurée en trois points au moyen de pyromètres à aspiration. La désulfuration est opérée dans un tube de $15 \mathrm{~cm}$ de diamètre et $4 \mathrm{~m}$ de hauteur. Les débits volumiques sont compris entre 100 et $200 \mathrm{Nm}^{3} / \mathrm{h}$, correspondant à des nombres de Reynolds supérieurs à 10000 .

L'écume de sucrerie est transportée vers le réacteur au moyen d'une pompe à suspension. Elle est introduite par l'intermédiaire d'un système de pulvérisation à air comprimé produisant des gouttelettes très fines, de manière à obtenir une évaporation rapide de l'eau de la suspension. Des mesures de température ont montré que le profil radial était plat $50 \mathrm{~cm}$ en

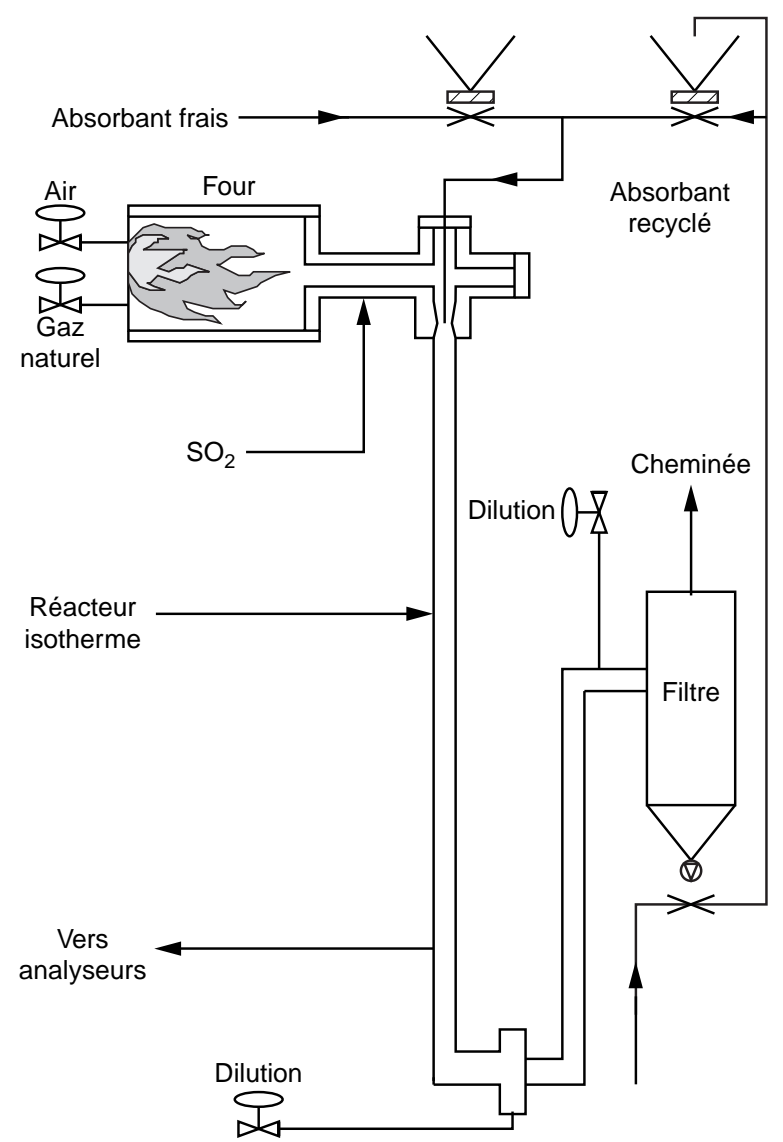

Figure 2

Réacteur $I F P$.

IFP Reactor

aval de l'injection, et que la température moyenne était alors atteinte. Ces mesures permettent de conclure que le temps caractéristique d'évaporation est faible devant le temps de séjour global des fumées dans le réacteur. L'échantillonnage des gaz est effectué par une sonde à aspiration. Cette sonde est refroidie par une circulation d'huile à $180^{\circ} \mathrm{C}$ pour prévenir les phénomènes de condensation en paroi.

\subsection{Analyse de l'écume de sucrerie}

L'échantillon utilisé pour cette étude a été fourni par la société Eridiana Beghin Say et provient de la sucrerie de Chevrières (France). L'analyse élémentaire en est donnée dans le tableau 1.

TABLEAU 1

Analyse des écumes de sucrerie

Carbonatation line analysis

\begin{tabular}{c|c|c|c|c|c|c|c|c|c}
\hline $\mathrm{Ca}(\%)$ & $\mathrm{Mg}$ & $\mathrm{C}$ & $\mathrm{H}$ & $\mathrm{O}$ & $\mathrm{N}$ & $\mathrm{Si}$ & $\mathrm{S}$ & $\mathrm{CaCO}_{3}$ & $\mathrm{MgCO}_{3}$ \\
\hline 32,5 & 1,3 & 13,2 & 0,7 & 29,2 & 0,5 & 1 & $<0,1$ & $81,3^{*}$ & $4,5^{*}$ \\
\hline
\end{tabular}

(*) valeurs calculées à partir de l'analyse élémentaire. 
Le magnésium ne participe pas à la capture du dioxyde de soufre car son sulfate est instable au-delà de $900^{\circ} \mathrm{C}$, dans les effluents de combustion [6]. Cependant, la décomposition du carbonate de magnésium peut accroître la porosité de la particule calcinée et favoriser ainsi sa perméabilité. La présence d'hydrogène, d'azote, de soufre et de carbone en excès est due à la matière organique résiduelle qui représente environ $5 \%$ de la masse, dans le cas présent. Le contenu organique des écumes de sucrerie apporte à la chaudière un surcroît de puissance, voisin de $2 \%$ de la puissance totale lorsque le rapport de débit molaire $\mathrm{Ca} / \mathrm{S}$ est égal à 2 . Les écumes de sucrerie contiennent environ $50 \%$ d'eau. La perte de rendement imputable à cette eau, environ $2 \%$ de la puissance de la chaudière, est en grande partie compensée par la combustion des matières organiques contenues dans les écumes.
La figure 3 permet de comparer l'aspect d'une écume de sucrerie séchée à celui d'un calcaire naturel broyé, caractéristique des produits que nous utilisons habituellement. Le calcaire naturel a un aspect homogène. Les faces des cristaux sont nettes et les particules ne sont pas poreuses. L'écume de sucrerie offre un aspect moins compact et les particules sont constituées d'un ensemble de grains élémentaires. La surface BET (Brunauer Emett Teller) a été trouvée comprise entre $10 \mathrm{~m}^{2} / \mathrm{g}$ et $20 \mathrm{~m}^{2} / \mathrm{g}$ pour tous les échantillons analysés, alors que celle du calcaire est égale à $2 \mathrm{~m}^{2} / \mathrm{g}$.

La répartition granulométrique de l'écume de sucrerie séchée est illustrée sur la figure 4 . La tranche 0-20 $\mu \mathrm{m}$ représente $90 \%$ de la masse. La finesse de la granulométrie facilite l'acheminement du $\mathrm{SO}_{2}$ à travers le réseau poreux de la particule calcinée et permet, dans une certaine mesure, de prévenir les limitations diffusionnelles. a

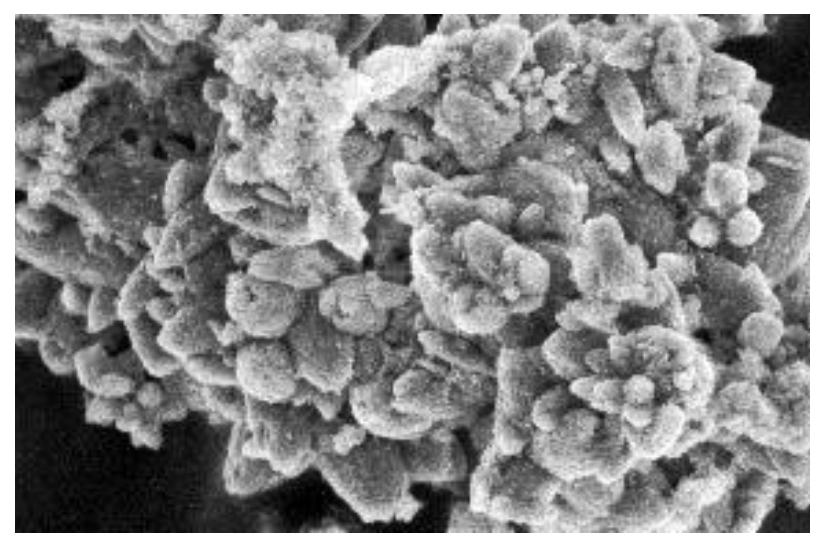

b

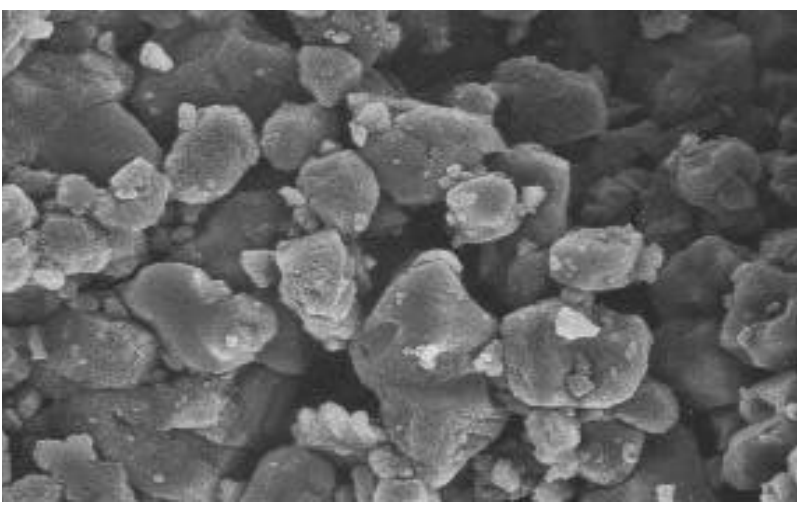

Figure 3

Comparaison entre une écume de sucrerie (a) et un calcaire naturel (b).

Comparison between carbonation line (a) and natural limestone (b).

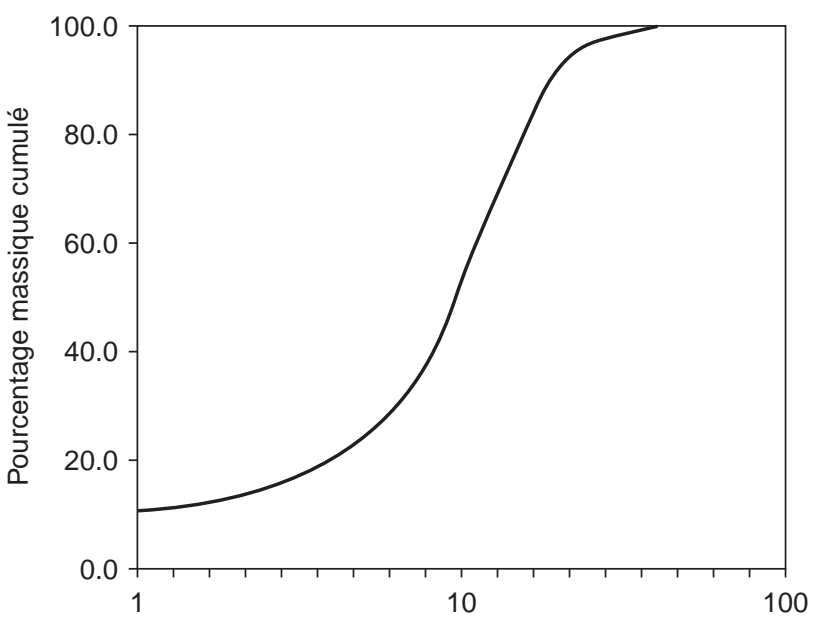

Figure 4

Répartition granulométrique de l'écume de sucrerie. Size distribution of carbonatation line.

\section{RÉSULTATS EXPÉRIMENTAUX}

Les essais ont été effectués dans les conditions opératoires suivantes :

- rapport de débit molaire $\mathrm{Ca} / \mathrm{S}$ : variable de 1 à 3 ;

- pression partielle en $\mathrm{SO}_{2}: 1400 \mathrm{ppm}$ et $2500 \mathrm{ppm}$;

- température : variable de $850{ }^{\circ} \mathrm{C}$ à $950{ }^{\circ} \mathrm{C}$;

- temps de séjour : $300 \mathrm{~ms}$.

Remarque : le temps de contact entre l'absorbant et les gaz est compris entre 1 et 2 secondes dans l'installation industrielle. Il n'est pas possible d'atteindre ces valeurs en laboratoire et les rendements de désulfuration ne sont donc pas représentatifs des performances du procédé. 


\subsection{Influence du rapport $\mathrm{Ca} / \mathrm{S}$ et de la pression partielle en $\mathrm{SO}_{2}$}

Sur la figure 5, le taux de désulfuration augmente de façon linéaire avec le rapport de débit molaire $\mathrm{Ca} / \mathrm{S}$. Le taux de sulfatation - calculé en base molaire - est donc constant et ne dépend pas de l'évolution de la concentration en $\mathrm{SO}_{2}$ dans le réacteur. Une augmentation significative du rendement de désulfuration est alors attendue avec un temps de séjour plus élevé. La figure 5 montre également que le rendement de désulfuration n'est pas modifié lorsque la pression partielle en $\mathrm{SO}_{2}$ passe de $2500 \mathrm{ppm}$ à $1400 \mathrm{ppm}$. Milne et al. [10] ont également observé que le taux de désulfuration était indépendant de la pression partielle en $\mathrm{SO}_{2}$ durant les premiers instants de la réaction, en travaillant avec du calcaire, de la chaux éteinte et des produits précalcinés. Ils ont également montré que l'ordre de la réaction devenait égal à 0,6 lorsque le taux de sulfatation était suffisant pour que la vitesse de réaction soit contrôlée par la diffusion du $\mathrm{SO}_{2}$ à travers la couche de sulfate en construction. La faible influence de la pression partielle en $\mathrm{SO}_{2}$ permet d'envisager une application du procédé de désulfuration à des installations brûlant des combustibles à très faible teneur en soufre, en cas de durcissement de la législation.

\subsection{Influence de la température}

La figure 6 indique que la température a une influence modérée sur le rendement de désulfuration. La variation du taux de sulfatation entre les deux températures extrêmes est proche de $5 \%$ et l'énergie d'activation apparente est voisine de
$9,5 \mathrm{kcal} / \mathrm{mol}$. La relative faiblesse de cette valeur peut indiquer l'intervention de limitations d'origines diffusionnelles. La faible influence de la température peut également être attribuée à un phénomène thermiquement activé qui provoque une diminution importante de la surface de réaction : le frittage.

Flament et al. [5] ont mesuré l'influence du frittage dans des conditions opératoires similaires. Ils ont alors montré que la surface développée lors de la calcination est deux fois plus faible à $1050^{\circ} \mathrm{C}$ qu'à $850^{\circ} \mathrm{C}$. Sur la figure 6 , le taux de sulfatation atteint $20 \%$ en $300 \mathrm{~ms}$, à $950^{\circ} \mathrm{C}$, et doit augmenter jusqu'à $1050^{\circ} \mathrm{C}$ d'après les résultats de Flament et al. [5].

\subsection{Comparaison avec un calcaire naturel et une chaux éteinte du commerce}

Il est communément admis que la chaux éteinte donne des rendements de désulfuration supérieurs à ceux obtenus avec le calcaire. Toutefois, le calcaire est généralement préféré pour des raisons économiques et peut être considéré comme le produit de référence. Les caractéristiques des absorbants utilisés sont données dans le tableau 2 et sur la figure 7.

TABLEAU 2

Analyse des absorbants calciques

Ca-based solvant analysis

\begin{tabular}{l|c|c|c|c|c}
\hline Absorbant & $\mathrm{Ca}(\%)$ & $\mathrm{Mg}(\%)$ & $\mathrm{Fe}(\%)$ & $\mathrm{S}(\%)$ & Surf. $\left(\mathrm{m}^{2} / \mathrm{g}\right)$ \\
\hline Calcaire & 38,6 & 0,6 & $<0,3$ & $<0,1$ & 2 \\
\hline Chaux éteinte & 52,1 & 1,4 & $<0,2$ & $<0,1$ & 20 \\
\hline
\end{tabular}

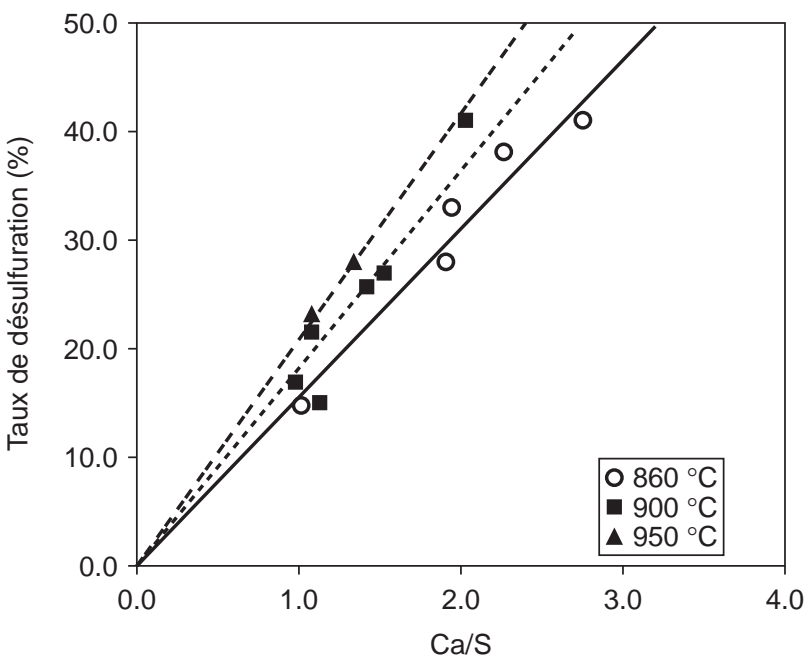

Figure 6

Influence de la température (absorbant : écume de sucrerie).

Temperature effect (carbonatation line).

Influence $\mathrm{du} \mathrm{Ca} / \mathrm{S}$ et de la pression partielle en $\mathrm{SO}_{2}$ 
Le calcaire naturel a été injecté sec et sous forme de suspension pour s'affranchir des éventuels effets de l'eau de l'écume. La figure 8 montre que l'écume de sucrerie donne des rendements de désulfuration supérieurs à ceux obtenus avec le calcaire naturel, quel que soit son mode d'injection. L'influence des propriétés initiales des absorbants sur la réactivité des particules calcinées n'est pas déterminée de manière claire. Cependant, Hartman et al. [3] ont remarqué que le taux de sulfatation était lié à la porosité du calcaire d'origine, résultat confirmé par d'autres travaux [7, 8]. D'après Borgwardt [9], le volume poreux initial de l'absorbant n'est pas détruit lors de la calcination et s'ajoute partiellement au volume poreux créé par le départ du $\mathrm{CO}_{2}$. L'oxyde de calcium produit par l'écume possède donc une porosité supérieure à celle de l'oxyde de calcium issu du calcaire naturel utilisé dans cette étude. En testant différentes origines de calcaire, Borgwardt a également remarqué que les produits constitués de micrograins, qui montraient des imperfections dans l'édifice cristallin, produisaient des oxydes de calcium mieux adaptés à la désulfuration que les produits parfaitement cristallisés.

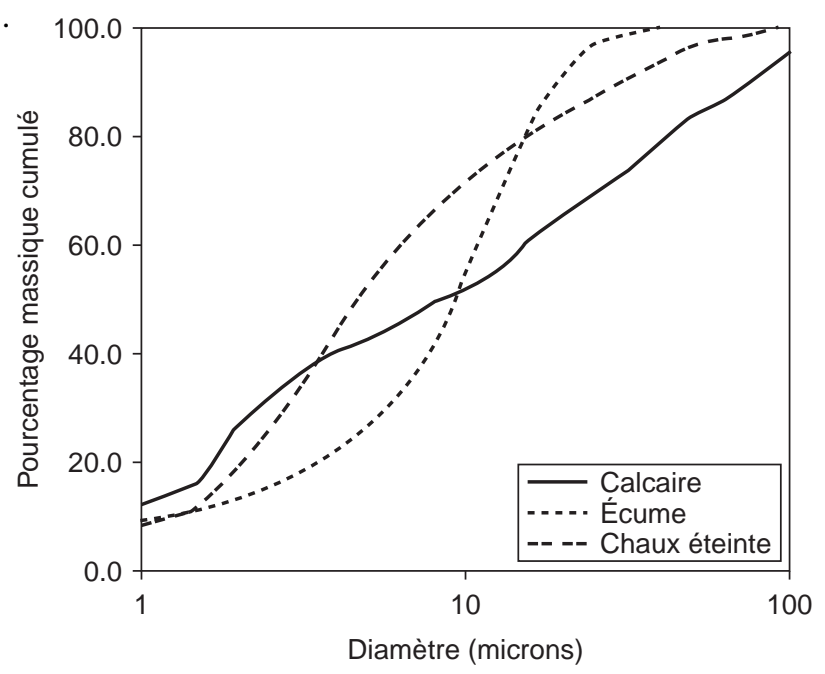

Figure 7

Répartition granulométrique des absorbants utilisés. Size distribution of sorbants.

\section{ESSAIS SUR CHAUDIĖRE SEMI-IN DUSTRIELLE (10 MW)}

Des essais ont été réalisés sur une chaudière industrielle de $10 \mathrm{MW}$ possédant tous les éléments caractéristiques de la chaudière Aude (fig. 9). L'absorbant usé peut être partiellement recyclé pour simuler au mieux la situation industrielle. Le temps de séjour dans la chambre de désulfuration est voisin de 1 seconde.
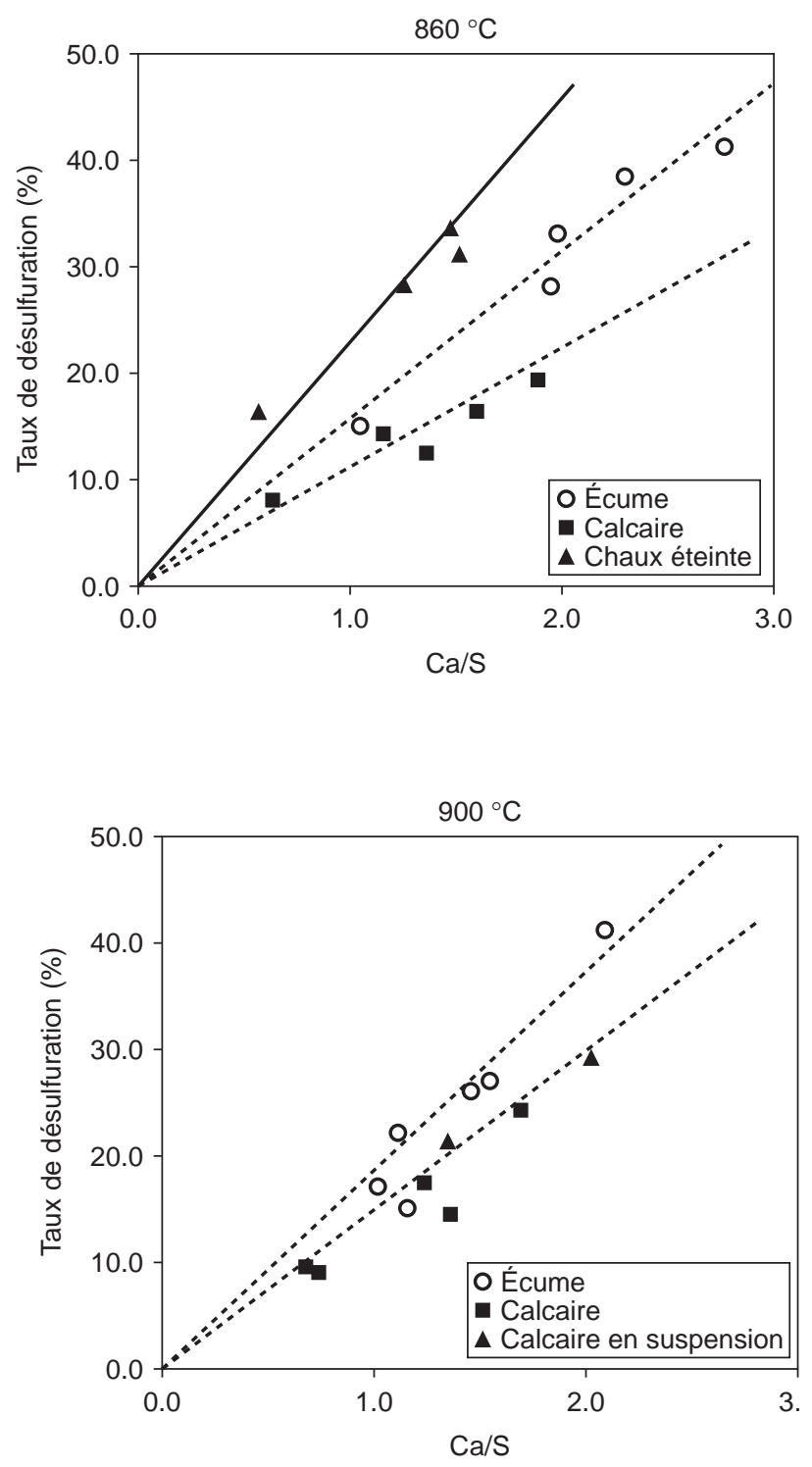

Figure 8

Évaluation de l'écume de sucrerie par rapport aux absorbants classiques

Comparison between carbonation line and common Ca-based sorbents.

\subsection{Désulfuration}

L'écume de sucrerie a été prélevée avant la mise en bassin de décantation. Des essais ont été effectués immédiatement, d'autres un an plus tard. Une partie de l'écume a donc été conservée en fût et nous avons pu regarder l'influence du vieillissement sur les performances de désulfuration. Le vieillissement modifie l'aspect de l'écume. Bien que les mesures de surface ne soient pas disponibles, la figure 10 montre que les écumes de fraîche production sont plus poreuses. 


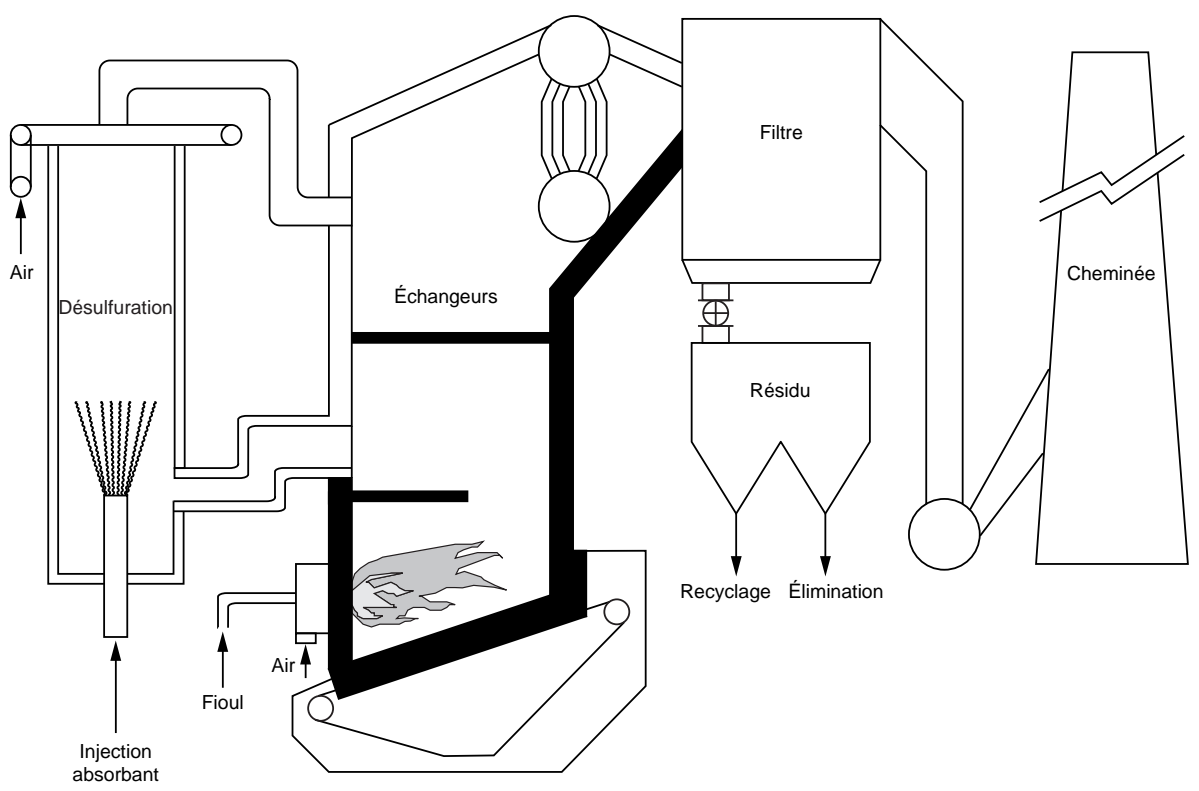

Figure 9

Chaudière semi-industrielle (10 MW).

Semi industrial boiler $(10 \mathrm{MW})$.

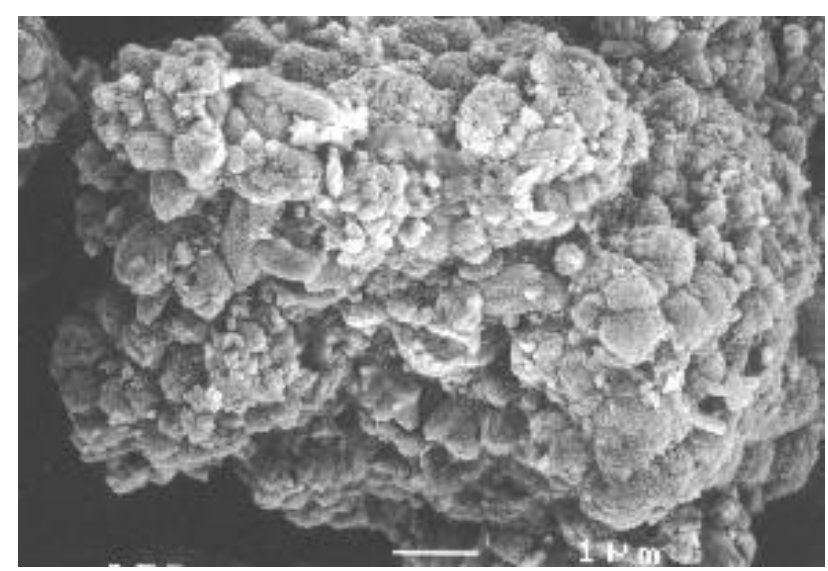

Figure 10

Comparaison entre écume "vieillie" (a) et écume "fraîche" (b).

Comparison between "fresh" (a) and "old" carbonatation line (b) . b

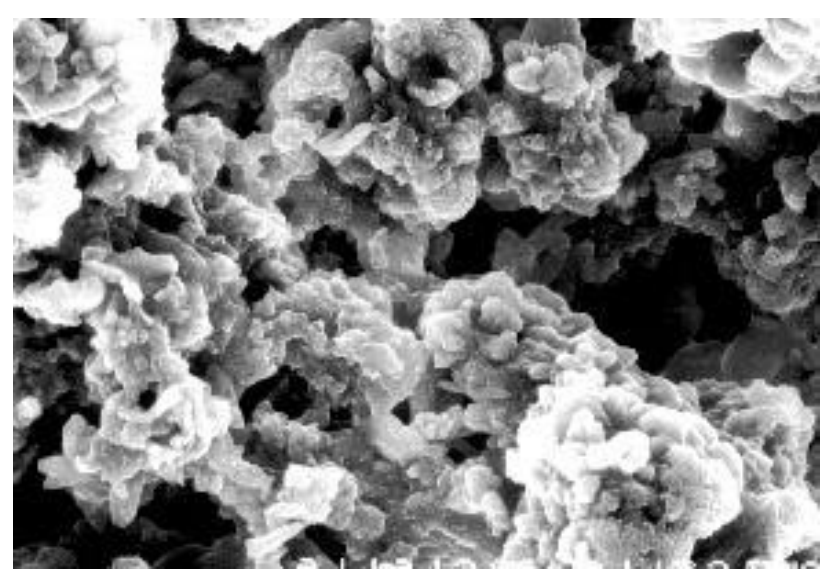

Les résultats obtenus sur la chaudière semi-industrielle confirment les conclusions tirées de l'étude en laboratoire. L'écume de sucrerie donne des rendements de désulfuration supérieurs à ceux obtenus avec un calcaire naturel (fig. 11). Ils peuvent être deux fois supérieurs en utilisant une écume de fraîche production. Le vieillissement de l'écume dégrade ses performances en diminuant la porosité d'après la figure 10. L'influence du recyclage a été étudiée avec le calcaire. Lorsque le débit d'absorbant recyclé est 3,5 fois plus élevé que le débit d'absorbant frais, un rendement de désulfuration supérieur à $70 \%$ peut être atteint avec un rapport $\mathrm{Ca} / \mathrm{S}$ égal à 1,7 . Un accroissement significatif du rendement est donc observé lorsque l'absorbant est recyclé. La disponibilité en écume était insuffisante pour effectuer le même type d'essai, en raison du temps nécessaire pour stabiliser l'installation. Toutefois, des performances supérieures sont attendues avec l'écume de sucrerie, sur la base des résultats de la figure 11. 


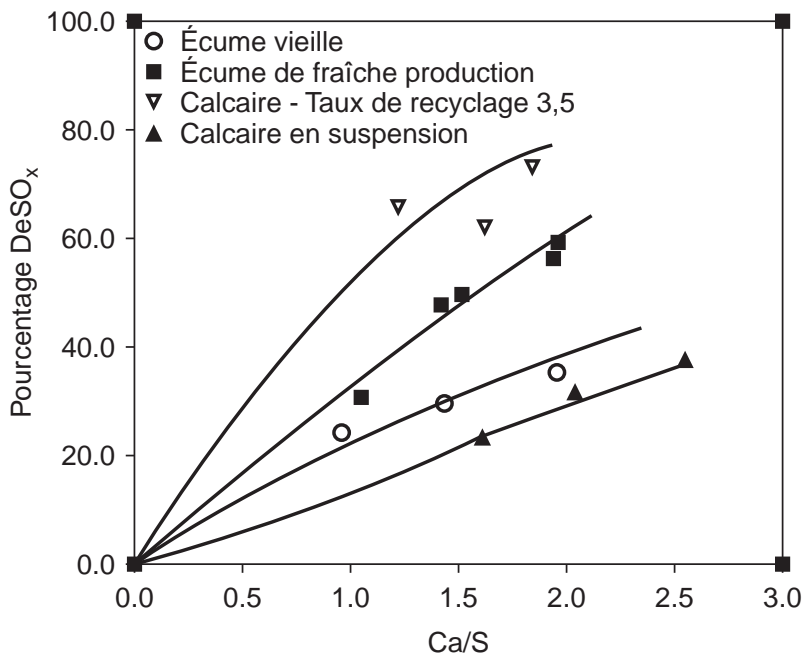

Figure 11

Désulfuration sur chaudière semi-industrielle.

Desulfurization in a semi industrial boiler.

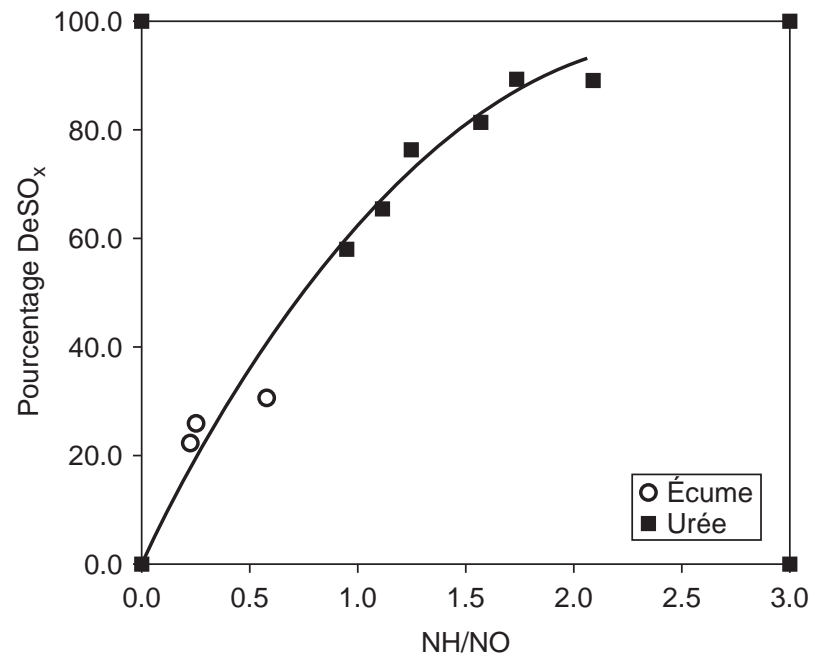

Figure 12

Réduction des oxydes d'azote par l'écume de sucrerie Carbonatation line effect on $\mathrm{NO}_{x}$ reduction.

\subsection{Dénitrification}

L'azote contenu par l'écume de sucrerie peut réduire les émissions d'oxyde d'azote. Des performances équivalentes à celles obtenues par injection d'urée ont été observées, comme le montre la figure 12. Ce résultat semble indiquer que l'écume de sucrerie est susceptible de réduire les $\mathrm{NO}_{\mathrm{x}}$ selon le mécanisme de la réduction sélective non catalytique [11]. En conditions classiques, c'est-à-dire avec un rapport $\mathrm{Ca} / \mathrm{S}$ égal à 2 , le rapport $\mathrm{NH} / \mathrm{NO}$ est voisin de 0,4 . La réduction des $\mathrm{NO}_{\mathrm{x}}$ atteint alors $20 \%$. Cependant, ces performances sont divisées par deux lorsque l'écume n'est pas de fraîche production, indiquant une évolution chimique des espèces azotées.

\section{BILAN ÉCONOMIQUE}

L'investissement est environ deux fois plus élevé pour une chaudière Aude que pour une chaudière brûlant du gaz naturel. Toutefois, les économies réalisées sur le prix du combustible permettent d'amortir cet investissement en moins de trois ans.

Hypothèses :

- consommation de fioul (4\% de soufre) : $60000 \mathrm{t} / \mathrm{a}$;

- consommation d'absorbant : 10300 t/a ;

- prix de l'absorbant : $500 \mathrm{~F} / \mathrm{t}$ (chaux hydratée) ;

- élimination de l'absorbant : $200 \mathrm{~F} / \mathrm{t}$;

- perte de rendement par rapport à une chaudière gaz : $2 \%$;

- électricité : $0,35 \mathrm{~F} / \mathrm{kWh}$;

- maintenance : $3 \%$;

- personnel : 1 demi-personne supplémentaire.
Le surcoût annuel d'exploitation est le suivant :

\begin{tabular}{l|l}
\hline absorbant & $5,2 \mathrm{MF}$ \\
élimination & $2,5 \mathrm{MF}$ \\
perte de rendement & $0,9 \mathrm{MF}$ \\
électricité & $0,4 \mathrm{MF}$ \\
maintenance & $0,8 \mathrm{MF}$ \\
personnel & $0,2 \mathrm{MF}$ \\
\hline surcoût annuel & $\mathbf{1 0 ~ M F}$ \\
\hline
\end{tabular}

Lorsque la teneur en $\mathrm{NO}_{\mathrm{x}}$ impose un traitement de fumées, il est nécessaire de rajouter environ $1 \mathrm{MF}$ au surcoût annuel d'exploitation, correspondant à la consommation d'urée. Le bilan économique du tableau 3 montre que le surcoût d'investissement par rapport à une chaudière brûlant du gaz, environ 35 MFF dans le cas présent, peut être payé en moins de trois ans. Cette situation correspond à une économie de 3,5 centimes/kWh sur le prix du combustible.

TABLEAU 3

Bilan économique

Economic balance

\begin{tabular}{l|c|c|c|c|c}
\hline Année & 1 & 2 & 3 & 4 & 5 \\
\hline Gain global & $13 \mathrm{MFF}$ & $26 \mathrm{MFF}$ & $39 \mathrm{MFF}$ & $52 \mathrm{MFF}$ & $65 \mathrm{MFF}$ \\
\hline
\end{tabular}

(gain global = gain sur combustible - surcoût d'exploitation)

La consommation d'absorbant représente la moitié du surcoût annuel d'exploitation. Ce surcoût peut donc être réduit de manière importante en utilisant des sous-produits industriels tels que l'écume de sucrerie. Si l'écume est mise à 
disposition gratuitement, et utilisée sur place, le temps de retour sur investissement devient alors voisin de deux ans. La teneur en eau élevée des écumes n'autorise pas un transport sur de longues distances. Sur la base d'un coût de transport égal à $0,80 \mathrm{~F} / \mathrm{km} / \mathrm{t}$, l'utilisation de l'écume de sucrerie n'est plus rentable à $200 \mathrm{~km}$ du point de production, par rapport à un calcaire pur à $90 \%$. Si l'écume est vendue $50 \mathrm{~F} / \mathrm{t}$, elle n'est plus rentable à $70 \mathrm{~km}$ du point de production, par rapport à un calcaire pur à $99 \%$. La teneur en eau est susceptible de limiter l'utilisation de l'écume de sucrerie, mais ce problème peut être résolu si la siccité est augmentée jusqu'à $70 \%$.

\section{CONCLUSION}

Des essais ont été effectués en laboratoire, en conditions bien contrôlées, pour obtenir des données de base sur la désulfuration par injection d'écume de sucrerie. Des tests de performance ont également été conduits sur une chaudière de $10 \mathrm{MW}$ pour vérifier que l'écume pouvait être utilisée en situation industrielle, avec des moyens d'injection appropriés. En conclusion, l'écume de sucrerie donne des rendements de désulfuration supérieurs à ceux obtenus avec un calcaire naturel, à répartition granulométrique comparable. Le contenu azoté de l'écume est également disponible pour réduire les oxydes d'azote selon le mécanisme de la réduction sélective non catalytique. L'utilisation de l'écume permet donc une économie sur la consommation d'urée ou d'ammoniaque lorsque la teneur en $\mathrm{NO}_{\mathrm{x}}$ doit être réduite de manière significative.

Le bilan économique montre que la chaudière Aude permet de brûler des produits à haute teneur en soufre, dans le respect de la législation, à un prix attractif. L'utilisation de l'écume de sucrerie diminue de façon significative le surcoût annuel d'exploitation. Cependant, la siccité de l'écume doit être voisine de $70 \%$ pour que son utilisation soit rentable sur de longues distances, par rapport au calcaire naturel.

\section{RÉFÉREN CES}

1 Martin, G. et Bouju, J.L. (1990) Procédé et dispositif pour générer de la chaleur comportant une désulfuration de effluents avec des particules d'absorbant de fine granulométrie en lit transporté. Brevet $\mathrm{n}^{\circ}$ 90/08311, FR2 664022 , IFP/Babcock Entreprise.

2 Kasaoka, S., Sakata, Y., Ito, M. et Ikeda, M. (1977) Reaction of Sulfur Dioxide with Various Metal Oxides and Thermal Cracking of Metal-Sulfate Products to Metal Oxides. Int. Chem. Eng., 17, 2.

3 Hartman, M., Svoboda, K. et Cermak, J. (1992) Sorption of Sulfur Dioxide by Waste Calcareous Marerial. Ind. Eng. Chem. Proc. Des. Dev., 21, 532-535.

4 Milne, C.R., Silcox, G.D., Pershing, D.W. et Kirchgessner, D.A. (1990) High-Temperature, Short-Time Sulfation of Calcium-Based Sorbents. 1. Theoritical Sulfation Model. Ind. Eng. Chem. Res., 29, 11, 2192.

5 Flament, P., Bortz, S. et Weber, R. (1987) Calcination and Sulfation Studies under Controlled Conditions for Direct Application to Desulfurization of Pulverized Coal Flames. IFRF Report on the $S 2$ Experiments, parties 1 et 2.

6 Flament, G. Direct Sulphur Capture in Flames through the Injection of Sorbents. IFRF Report, doc. $\mathrm{n}^{\circ} \mathrm{F}$ 038/a/1.

7 Bhatia, S.K., Perlmutter, D.D. (1981) AIChE J., 27, 226.

8 Dogu T. (1981) Chem. Eng. J., 21, 213.

9 Borgwardt, R.H. et Harvey, R.D. (1972) Environ. Sci. Technol. 6, 350.

10 Milne, C.R., Silcox, G.D., Pershing, D.W., Kirchgessner D.A. (1990) High-Temperature, Short-Time Sulfation of Calcium-Based Sorbents. 2. Experimental Data and Theoritical Model Predictions. Ind. Eng. Chem. Res., 29, 11, 2201.

11 Miller, J.A., Bowman, C.T. (1989) Mechanism and Modeling of Nitrogen Chemistry in Combustion. Prog. Energ. Comb. Sci., 15, 287. 